\title{
On the Exact Outage Probability of $2 \times 2$ MIMO-MRC in Correlated Rician Fading
}

\author{
Prathapasinghe Dharmawansa*, Kumara Kahatapitiya*, Saman Atapattu ${ }^{\ddagger}$, and Chintha Tellambura ${ }^{\S}$ \\ *Department of Electronic and Telecommunication Engineering, University of Moratuwa, Moratuwa, Sri Lanka \\ $\ddagger$ Department of Electrical and Electronic Engineering, University of Melbourne, Victoria, Australia \\ $\S$ Department of Electrical and Computer Engineering, University of Alberta, Edmonton, Canada
}

\begin{abstract}
This paper addresses a classical problem in random matrix theory-finding the distribution of the maximum eigenvalue of the correlated Wishart unitary ensemble. In particular, we derive a new exact expression for the cumulative distribution function (c.d.f.) of the maximum eigenvalue of a $2 \times 2$ correlated non-central Wishart matrix with rank- 1 mean. By using this new result, we derive the exact outage probability of $2 \times 2$ multipleinput multiple-output maximum-ratio-combining (MIMO-MRC) in Rician fading with transmit correlation and a strong line-ofsight (LoS) component (rank-1 channel mean). We also show that the outage performance is affected by the relative alignment of the eigen-spaces of the mean and correlation matrices. In general, when the LoS path aligns with the least eigenvector of the correlation matrix, in the high transmit signal-to-noise ratio (SNR) regime, the outage gradually improves with the increasing correlation. Moreover, we show that as $K$ (Rician factor) grows large, the outage event can be approximately characterized by the c.d.f. of a certain Gaussian random variable.
\end{abstract}

Index Terms-Maximum eigenvalue, MIMO-MRC, Noncentral Wishart matrix, Outage probability, Rician fading

\section{INTRODUCTION}

Multiple-input multiple-output (MIMO) systems are critical for $3 \mathrm{G}$ (third generation) and current $4 \mathrm{G}$ (fourth generation) wireless networks [2]. Traditional MIMO [3], [4] and virtual MIMO (e.g., cooperative relay networks [5]) are also a key enabler for 5G (fifth generation) and beyond wireless networks to accommodate the ever-increasing data demand. For example, in cell-free massive MIMO, where a large number of access points are distributed over a wide area, users and access point may have only one or two antennas [6]. Moreover, the simplest $2 \times 2$ MIMO channel becomes the most common channel and hence is of paramount importance for small-cell wireless networks [7]. For such small-size MIMO channels, accurate channel state information (CSI) estimation is fully viable, which enables the use of MIMO maximum-ratio-combining (MRC) receivers.

The performance of MIMO-MRC has been extensively analyzed for an arbitrary number of transmit and receive antennas for different channel models ( [8]-[12] and references therein). However, exact analytical characterization of the MIMO-MRC over the correlated Rician channel remains elusive. This challenge arises because the $2 \times 2$ correlated noncentral Wishart matrix does not admit analytically tractable joint eigenvalue density [13]. While general MIMO systems

This work is supported in part by the Australian Research Council (ARC) through the Discovery Early Career Researcher (DECRA) Award under Grant DE160100020.

A more detailed version of this paper can be found in [1]. are considered in the literature, only few studies have focused on small-scale practical MIMO architectures [14]-[17]. For instance, [14] derives the channel capacity of $2 \times 2$ or $2 \times 3$ MIMO channel for Nakagami- $m$ fading. In [15], the statistical properties of the Gram matrix $\mathbf{W}=\mathbf{H} \mathbf{H}^{\dagger}$, where $\mathbf{H}$ is a $2 \times 2$ complex central Gaussian matrix whose elements have arbitrary variances, have been investigated, resulting in exact distributions of $\mathbf{W}$ and its eigenvalues. In [16], the exact and asymptotic largest eigenvalue distributions of $\mathbf{W}$ are derived when $\mathbf{H}$ is a complex Gaussian matrix with unequal variances in the real and imaginary parts of its entries, or equivalently $\mathbf{H}$ belongs to the non-circularly-symmetric Gaussian subclass. These results have been then leveraged to analyze the outage performance of multi-antenna systems with MRC over Nakagami- $q$ (Hoyt) fading.

To the best of our knowledge, no exact performance analysis is available for the important case of $2 \times 2 \mathrm{MIMO}$ correlated Rician fading channel with rank-1 channel mean, i.e., a strong line-of-sight $(\mathrm{LoS})$ path exists between transmitter and receiver [18], [19]. To develop exact analytical results, we must first characterize the distribution of the maximum eigenvalue of the correlated non-central Wishart matrix. However, this distribution has remained an open problem in both wireless and even wider statistics literature. The main technical challenge is that the joint eigenvalue density of the correlated non-central Wishart matrix has no tractable representation. For instance, the invariant polynomial representation given in [13] cannot be integrated to obtain the marginal densities of the problem at hand. To circumvent this difficulty, here we follow an alternative approach [20], where the matrix variate density is directly integrated instead of the joint eigenvalue density, in order to find the maximum-eigenvalue distribution.

Specifically, we first derive an exact c.d.f. of the maximum eigenvalue of a $2 \times 2$ complex correlated none-central Wishart matrix with rank-1 mean, which is the key contribution of this paper. Some recent results on the maximum eigenvalue are symbolic, and not amenable to further processing, e.g., [12], [20]. We thus believe this to be the first tractable exact result on the maximum eigenvalue of such matrices. Subsequently, we also provide an exact expression for the outage probability. Additionally, we also characterize the effect of the Rician factor $K$ on the outage probability for different signal-to-noise ratio (SNR) regimes and establish stochastic convergence limit of outage probability for large $K$ values.

The following notation is used throughout this paper. The superscript $(\cdot)^{\dagger}$ indicates the Hermitian-transpose, and $(\cdot)^{T}$ 
stands for the matrix transpose. We use $\operatorname{det}(\cdot)$ to represent the determinant of a square matrix, $\operatorname{tr}(\cdot)$ to represent trace, and $\operatorname{etr}(\cdot)$ stands for $\exp (\operatorname{tr}(\cdot))$. Positive definiteness of a square matrix $\mathbf{A}$ is represented by $\mathbf{A} \succ \mathbf{0}$, and $\mathbf{A} \succ \mathbf{B}$ denotes $\mathbf{A}-\mathbf{B} \succ \mathbf{0}$. The square root of a positive definite matrix $\mathbf{G}$ is denoted by $\mathbf{G}^{\frac{1}{2}}$ and $\operatorname{diag}\left\{s_{1}, s_{2}\right\}$ denotes a $2 \times 2$ diagonal matrix with the real diagonal entries $s_{1}$ and $s_{2}$. We use $\lambda_{\max }(\mathbf{A})$ to denote the maximum eigenvalue of a square matrix $\mathbf{A}$. The real and imaginary parts, modulus and conjugate of a complex number $z$ are denoted, respectively, by $\Re(z), \Im(z),|z|$ and $z^{*}$. The Euclidean norm of a vector $\mathbf{w}$ is denotes by $\|\mathbf{w}\| .\lfloor x\rfloor$ denotes the floor function, defined as $\lfloor x\rfloor=\max \{m \in \mathbb{Z} \mid m \leq x\}$. Finally, the union of two measurable sets $\mathcal{R}_{1}$ and $\mathcal{R}_{2}$ is denoted by $\mathcal{R}_{1} \cup \mathcal{R}_{2}$.

\section{CDF of The Maximum Eigenvalue}

This section derives the new expression for the distribution of the maximum eigenvalue of a $2 \times 2$ correlated complex non-central Wishart matrix with rank-1 mean matrix. Before proceeding with the derivations, we present some fundamental statistical characteristics of the complex correlated non-central Wishart matrix.

Definition 1. Let $\mathbf{X}$ be an $n \times m(n \geq m)$ complex Gaussian random matrix distributed as $\mathcal{C N}_{n, m}\left(\mathbf{\Upsilon}, \mathbf{I}_{n} \otimes \mathbf{\Psi}\right)$, where $\boldsymbol{\Psi} \in \mathbb{C}^{m \times m} \succ \mathbf{0}$ and $\mathbf{\Upsilon} \in \mathbb{C}^{n \times m}$. Then $\mathbf{W}=\mathbf{X}^{\dagger} \mathbf{X}$ has a complex non-central Wishart distribution $\mathcal{W}_{m}(n, \mathbf{\Psi}, \boldsymbol{\Theta})$ with the density function [21]

$$
\begin{aligned}
f(\mathbf{W})= & \frac{\operatorname{etr}(-\boldsymbol{\Theta}) \operatorname{det}^{n-m}(\mathbf{W})}{\tilde{\Gamma}_{m}(n) \operatorname{det}^{n}(\mathbf{\Psi})} \\
& \operatorname{etr}\left(-\mathbf{\Psi}^{-1} \mathbf{W}\right)_{0} \tilde{F}_{1}\left(n ; \boldsymbol{\Theta} \mathbf{\Psi}^{-1} \mathbf{W}\right)
\end{aligned}
$$

where $\Theta=\Psi^{-1} \mathbf{\Upsilon}^{\dagger} \mathbf{\Upsilon}$ is the non-centrality parameter, ${ }_{0} \tilde{F}_{1}(\cdot ; \cdot)$ denotes Bessel type complex hypergeometric function of matrix argument and the complex multivariate gamma function is defined as $\tilde{\Gamma}_{m}(n) \triangleq \pi^{\frac{m(m-1)}{2}} \prod_{j=1}^{m} \Gamma(n-j+1)$ with $\Gamma(\cdot)$ being the gamma function.

Next we define the joint eigenvalue distribution of $\mathbf{W}$ which consists of invariant polynomials due to Davis [22], [23].

Corollary 1. The joint density of ordered eigenvalues $\lambda_{1}>$ $\lambda_{2}>\ldots . .>\lambda_{m}>0$, of the complex non-central Wishart matrix $\mathbf{W}$ is given by [13, Eq. 5.4]

$$
\begin{aligned}
g(\boldsymbol{\Lambda})= & \frac{\pi^{m(m-1)} \operatorname{etr}(-\Theta)}{\tilde{\Gamma}_{m}(n) \tilde{\Gamma}_{m}(m) \operatorname{det}^{n}(\boldsymbol{\Psi})} \prod_{k=1}^{m} \lambda_{k}^{n-m} \prod_{k<l}\left(\lambda_{k}-\lambda_{l}\right)^{2} \\
& \times \sum_{k, t=0}^{\infty} \sum_{\kappa, \tau ; \phi \in \kappa . \tau} \frac{C_{\phi}^{\kappa, \tau}\left(-\boldsymbol{\Psi}^{-1}, \boldsymbol{\Theta} \boldsymbol{\Psi}^{-1}\right) C_{\phi}^{\kappa, \tau}(\boldsymbol{\Lambda}, \boldsymbol{\Lambda})}{k ! t ![n]_{\tau} C_{\phi}\left(\mathbf{I}_{m}\right)}
\end{aligned}
$$

where $\boldsymbol{\Lambda}$ is the diagonal matrix having eigenvalues of $\mathbf{W}$ along the main diagonal, $C_{\phi}^{\kappa, \tau}(\cdot, \cdot)$ denotes an invariant polynomial with two matrix arguments [22], [23] and the complex hypergeometric coefficient $[n]_{\tau}$ is defined as $[n]_{\tau}=$ $\prod_{j=1}^{m}(n-j+1)_{\tau_{j}}$ in which $\tau=\left(\tau_{1}, \tau_{2}, \ldots, \tau_{m}\right)$ is a partition of the integer $t$ into $m$ parts such that $\sum_{j=1}^{m} \tau_{j}=t$ and $\tau_{1} \geq \tau_{2} \geq \ldots . \tau_{m} \geq 0$. Also, $(a)_{k}$ is the Pochhammer symbol given by $(a)_{k}=\frac{\overline{\Gamma(a+k)}}{\Gamma(a)}$ with $(a)_{0}=1$.

\section{A. Cumulative Distribution Function of the Maximum Eigen- value}

Here, to derive the c.d.f. of the maximum eigenvalue, the most straightforward method is to find the probability that the interval $[x, \infty)$ is free from the eigenvalues [24]-[27]. As such, we may write, $F_{\lambda_{\max }}(x)=\int_{\mathcal{D}} g(\boldsymbol{\Lambda}) \mathrm{d} \lambda_{1} \ldots \mathrm{d} \lambda_{\mathrm{m}}$, where $\mathcal{D}=\left\{0<\lambda_{m}<\ldots<\lambda_{1}<x\right\}$. This direct method, however, is cumbersome because of the invariant polynomials in eq (2). Despite their theoretical significance and frequent appearance in multivariate distribution theory, the invariant polynomials do not seem to admit simple forms in terms of the eigenvalues of its argument matrices even for the $2 \times 2$ case [22], [23]. To circumvent this difficulty, we will adopt an alternative approach based on integrating directly over matrix variate distribution, instead of the joint eigenvalue distribution [13], [20], [22], [28]-[30]. More precisely, we write the c.d.f. of the maximum eigenvalue as

$$
F_{\lambda_{\max }}(x)=\operatorname{Pr}\left(\mathbf{W} \prec x \mathbf{I}_{m}\right)
$$

which facilitates the use of the probability density function of $\mathbf{W}$ instead of its eigenvalue distribution. Now we apply the change of variable $\mathbf{W}=x \mathbf{Z}$, where $\mathbf{Z}$ is Hermitian positive definite with $\mathrm{d} \mathbf{W}=x^{m^{2}} \mathrm{~d} \mathbf{Z}$, in (3) to yield

$$
\begin{aligned}
F_{\lambda_{\max }}(x)= & \frac{x^{m n} \operatorname{etr}(-\mathbf{\Theta})}{\tilde{\Gamma}_{m}(n) \operatorname{det}^{n}(\mathbf{\Psi})} \int_{\mathbf{0} \prec \mathbf{Z} \prec \mathbf{I}_{m}} \operatorname{det}^{n-m}(\mathbf{Z}) \\
& \quad \operatorname{etr}\left(-x \mathbf{\Psi}^{-1} \mathbf{Z}\right)_{0} \tilde{F}_{1}\left(n ; x \boldsymbol{\Theta} \Psi^{-1} \mathbf{Z}\right) \mathrm{d} \mathbf{Z} .
\end{aligned}
$$

As shown in [13] and [20], this integral does not admit simple form for arbitrary values of $m$ and $n$ even for rank one mean matrix. However, as we now show, it can be solved in terms of simple functions for the important configuration of $m=$ $n=2$. $^{1}$

In the case of $m=n=2$, (4) simplifies to

$$
\begin{gathered}
F_{\lambda_{\max }}(x)=\frac{x^{4} \exp (-\eta)}{\pi \operatorname{det}^{2}(\mathbf{\Psi})} \int_{\mathbf{0} \prec \mathbf{Z} \prec \mathbf{I}_{2}} \operatorname{etr}\left(-x \boldsymbol{\Psi}^{-1} \mathbf{Z}\right) \\
{ }_{0} \tilde{F}_{1}\left(2 ; x \boldsymbol{\Theta} \Psi^{-1} \mathbf{Z}\right) \mathrm{d} \mathbf{Z}
\end{gathered}
$$

where $\eta=\operatorname{tr}(\boldsymbol{\Theta})$. Observing the fact that the matrix $\boldsymbol{\Psi}$ is Hermitian positive definite having the eigen-decomposition $\boldsymbol{\Psi}=\mathbf{U} \boldsymbol{\Omega} \mathbf{U}^{\dagger}$, where $\mathbf{U} \in \mathbb{C}^{2 \times 2}$ is unitary and $\boldsymbol{\Omega}=$ $\operatorname{diag}\left\{\omega_{1}, \omega_{2}\right\}$ with $\omega_{1} \geq \omega_{2}>0$, we can rewrite (5) with the help of the transformation $\mathbf{Y}=\mathbf{U}^{\dagger} \mathbf{Z U}$ as

$$
\begin{gathered}
F_{\lambda_{\max }}(x)=\frac{x^{4}}{\pi} \operatorname{etr}(-\eta)\left(\sigma_{1} \sigma_{2}\right)^{2} \int_{\mathbf{0} \prec \mathbf{Y} \prec \mathbf{I}_{2}} \operatorname{etr}(-x \boldsymbol{\Sigma} \mathbf{Y}) \\
{ }_{0} \tilde{F}_{1}\left(2 ; x \boldsymbol{\Sigma} \mathbf{U}^{\dagger} \mathbf{\Upsilon}^{\dagger} \mathbf{\Upsilon} \mathbf{U} \boldsymbol{\Sigma} \mathbf{Y}\right) \mathrm{d} \mathbf{Y}
\end{gathered}
$$

where $\boldsymbol{\Sigma}=\boldsymbol{\Omega}^{-1}=\operatorname{diag}\left\{\sigma_{1}, \sigma_{2}\right\}$ and $0<\sigma_{1} \leq \sigma_{2}$. Observing the fact that $\boldsymbol{\Sigma} \mathbf{U}^{\dagger} \boldsymbol{\Upsilon}^{\dagger} \boldsymbol{\Upsilon} \mathbf{U} \boldsymbol{\Sigma}$ is Hermitian non-negative definite with rank one, it can be expressed via its eigen-decompsotion

\footnotetext{
${ }^{1}$ Although our general approach is valid even for $n>2$, here we focus on $n=2$ in view of obtaining a relatively not so complicated answer.
} 


$$
\begin{aligned}
\mathcal{I}_{k}(x)=\sum_{p=0}^{\left\lfloor\frac{k}{2}\right\rfloor} \sum_{j=0}^{k-2 p} \frac{a_{1}(k, p, j)}{\left(x \sigma_{2}\right)^{j+p+2}}(j+p+1) !\left[\frac{{ }_{1} F_{1}\left(1 ; c_{k, p, j}+3 ; x \sigma_{1}\right)}{\left(c_{k, p, j}+2\right)}-\sum_{i=0}^{j+p+1} \frac{\left(c_{k, p, j}+1\right) !}{\left(c_{k, p, j}+i+2\right) !}\left(x \sigma_{2}\right)^{i}\right. & \left.\times{ }_{1} F_{1}\left(i+1 ; c_{k, p, j}+i+3 ; x\left(\sigma_{1}-\sigma_{2}\right)\right)\right], \\
\mathcal{J}_{k}(x)= & \sum_{p=0}^{\left\lfloor\frac{k}{2}\right\rfloor} \sum_{j=0}^{k-2 p}\left[a_{2}(k, p, j) \exp \left(-x \sigma_{2}\right)_{1} F_{1}\left(p+2 ; c_{k, p, j}+3 ; x \sigma_{1}\right){ }_{1} F_{1}\left(p+2 ; j+p+3 ; x \sigma_{2}\right)\right. \\
& \left.+\sum_{l=0}^{p+1} \sum_{q=0}^{j+l} \frac{a_{3}(k, p, j, l, q)}{\left(x \sigma_{2}\right)^{j+l+1-q}}{ }_{1} F_{1}\left(p+q+2 ; c_{k, p, j}+q+3 ; x\left(\sigma_{1}-\sigma_{2}\right)\right)-\sum_{l=0}^{p+1} \frac{a_{4}(k, p, j, l)}{\left(x \sigma_{2}\right)^{j+l+1}}{ }_{1} F_{1}\left(p+2 ; c_{k, p, j}+3 ; x \sigma_{1}\right)\right]
\end{aligned}
$$

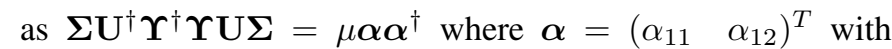
$\alpha_{11}, \alpha_{12} \in \mathbb{C}, \mu>0$ and $\boldsymbol{\alpha}^{\dagger} \boldsymbol{\alpha}=1$. This in turn gives the relation $\mu=\operatorname{tr}\left(\boldsymbol{\Theta} \Psi^{-1}\right)$. Therefore, we can rewrite (6) as

$$
\begin{aligned}
F_{\lambda_{\max }}(x)= & \frac{x^{4}}{\pi} \operatorname{etr}(-\eta)\left(\sigma_{1} \sigma_{2}\right)^{2} \\
& \int_{\mathbf{0} \prec \mathbf{Y} \prec \mathbf{I}_{2}} \operatorname{etr}(-x \boldsymbol{\Sigma} \mathbf{Y})_{0} \tilde{F}_{1}\left(2 ; x \mu \boldsymbol{\alpha} \boldsymbol{\alpha}^{\dagger} \mathbf{Y}\right) \mathrm{d} \mathbf{Y} .
\end{aligned}
$$

Further manipulation in this form is not desirable. However, we expand the hypergeometric function with its equivalent zonal series expansion to yield [21]

$$
\begin{aligned}
F_{\lambda_{\max }}(x)= & \frac{x^{4}}{\pi} \operatorname{etr}(-\eta)\left(\sigma_{1} \sigma_{2}\right)^{2} \sum_{k=0}^{\infty} \sum_{\kappa} \frac{(x \mu)^{k}}{[2]_{\kappa} k !} \\
& \int_{\mathbf{0} \prec \mathbf{Y} \prec \mathbf{I}_{2}} \operatorname{etr}(-x \boldsymbol{\Sigma} \mathbf{Y}) C_{\kappa}\left(\boldsymbol{\alpha} \boldsymbol{\alpha}^{\dagger} \mathbf{Y}\right) \mathrm{d} \mathbf{Y}
\end{aligned}
$$

where $C_{\kappa}(\cdot)$ is the zonal polynomial in which $\kappa=\left(\kappa_{1}, \kappa_{2}\right)$ represents a partition of $k$ into not more than two parts such that $\kappa_{1} \geq \kappa_{2} \geq 0$ [21], [31]. Since the matrix $\boldsymbol{\alpha} \boldsymbol{\alpha}^{\dagger} \mathbf{Y}$ is rank one, we have $C_{\kappa}\left(\boldsymbol{\alpha} \boldsymbol{\alpha}^{\dagger} \mathbf{Y}\right)=0$ for all partitions $\kappa$ having more than one non-zero parts [31]. Therefore, the zonal polynomial degenerates to $C_{\kappa}\left(\boldsymbol{\alpha} \boldsymbol{\alpha}^{\dagger} \mathbf{Y}\right)=\left(\boldsymbol{\alpha}^{\dagger} \mathbf{Y} \boldsymbol{\alpha}\right)^{k}$. Hence we can simplify (7) to obtain

$$
F_{\lambda_{\max }}(x)=\frac{x^{4}}{\pi} \operatorname{etr}(-\eta)\left(\sigma_{1} \sigma_{2}\right)^{2} \sum_{k=0}^{\infty} \frac{(x \mu)^{k}}{(2)_{k} k !} Q_{k}(x)
$$

where

$$
Q_{k}(x)=\int_{\mathbf{0} \prec \mathbf{Y} \prec \mathbf{I}_{2}} \operatorname{etr}(-x \boldsymbol{\Sigma} \mathbf{Y})\left(\boldsymbol{\alpha}^{\dagger} \mathbf{Y} \boldsymbol{\alpha}\right)^{k} \mathrm{~d} \mathbf{Y} .
$$

This integral does not seem to have a simple solution in terms of simple functions according to the literature [13], [21]. Therefore, in the sequel, we demonstrate how to evaluate this integral in terms of hypergeometric functions which in turn yield an exact expression for the c.d.f. of the maximum eigenvalue.

The theorem below gives the c.d.f. of the maximum eigenvalue of a $2 \times 2$ non-central Wishart matrix with two degrees of freedom.

Theorem 1. Let $\mathbf{X}$ be a $2 \times 2$ complex square matrix

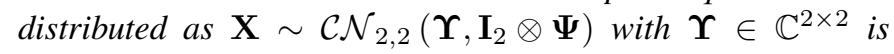

rank one. Then the c.d.f. of the maximum eigenvalue $\lambda_{\max }$ of the semi-correlated non-central complex Wishart matrix $\mathbf{W}=\mathbf{X}^{\dagger} \mathbf{X}$ is given by

$$
\begin{aligned}
F_{\lambda_{\max }}(x)=\left(\sigma_{1} \sigma_{2}\right)^{2} & x^{4} \exp \left(-\sigma_{1} x-\eta\right) \\
& \times \sum_{k=0}^{\infty} \frac{(x \mu)^{k}}{(k+1) !}\left[\mathcal{I}_{k}(x)+\mathcal{J}_{k}(x)\right]
\end{aligned}
$$

where $\mathcal{I}_{k}(x)$ and $\mathcal{J}_{k}(x)$ are given on top of the page with

$$
\begin{aligned}
& a_{1}(k, p, j)=\frac{\left|\alpha_{11}\right|^{2 c_{k, p, j}}\left|\alpha_{12}\right|^{2(p+j)}}{j ! p !(p+1) !\left(c_{k, p, j}-p\right) !} \\
& a_{2}(k, p, j)=\frac{(p+1)\left|\alpha_{11}\right|^{2 c_{k, p, j}}\left|\alpha_{12}\right|^{2(p+j)}}{(j+p+2) !\left(c_{k, p, j}+2\right) !} \\
& a_{3}(k, p, j, l, q)=\frac{(-1)^{l}(p+1)(j+l) !\left|\alpha_{11}\right|^{2 c_{k, p, j}}\left|\alpha_{12}\right|^{2(p+j)}}{j ! l !(p+1-l) !\left(c_{k, p, j}+2\right) !} \\
& a_{4}(k, p, j, l)=\frac{(-1)^{l}(j+l) !(p+q+1) !\left|\alpha_{11}\right|^{2 c} c_{k, p, j}\left|\alpha_{12}\right|^{2(p+j)}}{j ! ! ! p ! q !(p+1-l) !\left(c_{k, p, j}+q+2\right) !}
\end{aligned}
$$

and $c_{k, p, j}=k-p-j$. Also, ${ }_{1} F_{1}(\cdot ; \cdot ; \cdot)$ denotes the confluent hypergeometric function of the first kind, $\boldsymbol{\Theta}=\boldsymbol{\Psi}^{-1} \boldsymbol{\Upsilon}^{\dagger} \boldsymbol{\Upsilon}$, $\boldsymbol{\Psi}=\mathbf{U} \boldsymbol{\Omega} \mathbf{U}^{\dagger}, \mu=\operatorname{tr}\left(\boldsymbol{\Theta} \boldsymbol{\Psi}^{-1}\right), \eta=\operatorname{tr}(\boldsymbol{\Theta}), \boldsymbol{\Sigma}=\boldsymbol{\Omega}^{-1}=$ $\operatorname{diag}\left\{\sigma_{1}, \sigma_{2}\right\}, \boldsymbol{\Sigma} \mathbf{U}^{\dagger} \mathbf{\Upsilon}^{\dagger} \boldsymbol{\Upsilon} \mathbf{U} \boldsymbol{\Sigma}=\mu \boldsymbol{\alpha} \boldsymbol{\alpha}^{\dagger}$, and $\boldsymbol{\alpha}=\left(\alpha_{11} \alpha_{12}\right)^{T}$.

Proof. See Appendix A.

It is remarkable that the above c.d.f. expression depends on, among other parameters, the components of the eigenvector of the rank one matrix $\boldsymbol{\Sigma} \mathbf{U}^{\dagger} \boldsymbol{\Upsilon}^{\dagger} \boldsymbol{\Upsilon} \mathbf{U} \boldsymbol{\Sigma}$, in contrast to corresponding expressions for correlated Rayleigh and uncorrelated Rician matrices which do not depend on the eigenvectors [26]. This represents the joint effect of correlation and mean. As a simple sanity check of Theorem 1, Fig. 1 shows the comparison of theoretical c.d.f. expression (10) and simulation results for the following parameters:

$$
\begin{aligned}
\mathbf{\Upsilon} & =\left(\begin{array}{cc}
1.0000+0.0000 i & 0.3624-0.9320 i \\
0.8878-0.4603 i & -0.1073-0.9942 i
\end{array}\right) \\
\Psi & =\left(\begin{array}{cc}
1.0000+0.0000 i & -0.3731-0.4902 i \\
-0.3731+0.4902 i & 1.0000+0.0000 i
\end{array}\right) .
\end{aligned}
$$

It is also noteworthy that the infinite series has been truncated to a maximum of 15 terms; thereby demonstrating a fast convergence rate for each case. 


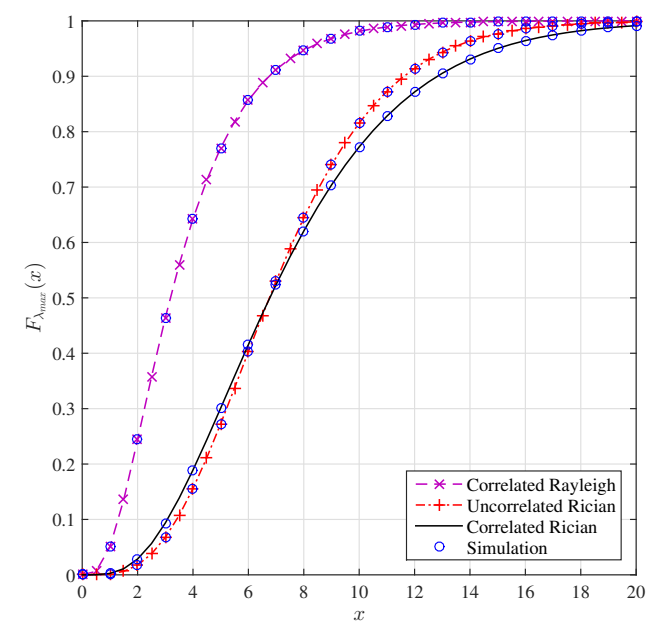

Fig. 1: Outage probability vs normalized SNR threshold $\gamma_{\text {th }} / \bar{\gamma}$ for correlated Rayleigh, uncorrelated/correlated Rician without $K$ normalization.

\section{PERFORMANCE OF $2 \times 2$ MIMO BEAMFORMING}

To emphasize the utility of Theorem 1 , here we focus on the performance of $2 \times 2$ MIMO MRC over a correlated Rician fading channel. In particular, we analyze an important performance metric - outage probability.

Consider the following $2 \times 2$ MIMO Rician channel model [11], [12]

$$
\mathbf{H}=\sqrt{\frac{K}{K+1}} \overline{\mathbf{H}}+\sqrt{\frac{1}{K+1}} \mathbf{H}_{s c} \mathbf{T}^{\frac{1}{2}}
$$

where $\overline{\mathbf{H}} \in \mathbb{C}^{2 \times 2}$ is the deterministic component, $\mathbf{H}_{s c} \sim$ $\mathcal{C N}_{2,2}\left(\mathbf{0}_{2 \times 2}, \mathbf{I}_{2} \otimes \mathbf{I}_{2}\right)$ represents the Rayleigh random component, and $\mathbf{T} \in \mathbb{C}^{2 \times 2} \succ \mathbf{0}$ is the transmit correlation matrix with $K$ being the Rician factor. Moreover, we adapt the common normalization used in the literature to suit our requirement as $\operatorname{tr}\left(\overline{\mathbf{H}}^{\dagger} \overline{\mathbf{H}}\right)=4$ and $\operatorname{tr}(\mathbf{T})=2$. It is noteworthy that in the presence of a strong LoS path between the transmitter and receiver (i.e., Rician fading), the rank of the matrix $\overline{\mathbf{H}}$ degenerates to one [11], [12]. Therefore, here we focus on the rank-1 $\overline{\mathbf{H}}$ case only. Since $\mathbf{H}$ is a complex Gaussian random matrix, following Definition $1, \mathbf{W}=\mathbf{H}^{\dagger} \mathbf{H}$ is correlated complex non-central Wishart distributed. Therefore, the corresponding covariance and the non-centrality parameter matrices can be written, respectively, as $\boldsymbol{\Psi}=\left(\frac{1}{K+1}\right) \mathbf{T}$ and $\boldsymbol{\Theta}=\boldsymbol{\Psi}^{-1} \boldsymbol{\Upsilon}^{\dagger} \boldsymbol{\Upsilon}=K \mathbf{T}^{-1} \overline{\mathbf{H}}^{\dagger} \overline{\mathbf{H}}$.

Now consider a point-to-point MIMO link with two transmit and two receive antennas. The received information vector $\mathbf{r} \in$ $\mathbb{C}^{2 \times 1}$ is given by

$$
\mathbf{r}=\sqrt{P} \mathbf{H w} s+\mathbf{n}
$$

where the channel $\mathbf{H} \in \mathbb{C}^{2 \times 2}$ is given by (12) with $\overline{\mathbf{H}}$ being rank- $1, P$ is the transmit power, $\mathbf{w} \in \mathbb{C}^{2 \times 1}$ is the beamforming vector with $\|\mathbf{w}\|^{2}=1, s \in \mathbb{C}$ is the transmitted symbol with
$\mathbb{E}\left\{|s|^{2}\right\}=1$, and $\mathbf{n} \in \mathbb{C}^{2 \times 1}$ is the additive white Gaussian noise (AWGN) vector with zero mean and $N_{0} \mathbf{I}_{2}$ covariance matrix. We assume that both transmitter and the receiver have perfect instantaneous CSI.

A MIMO-MRC receiver determines $w$ such that the received SNR, $\gamma=\bar{\gamma} \mathbf{w}^{\dagger} \mathbf{H}^{\dagger} \mathbf{H} \mathbf{w}$ is maximized. Here $\bar{\gamma}=P / N_{0}$ denotes the transmit SNR. It is well documented that the vector w which maximizes $\gamma$ is the leading eigenvector of $\mathbf{H}^{\dagger} \mathbf{H}$ [8], [32]. Therefore, the maximum received SNR is given by

$$
\gamma=\bar{\gamma} \lambda_{\max }
$$

where $\lambda_{\max }$ is the leading eigenvalue of $\mathbf{H}^{\dagger} \mathbf{H}$. This clearly demonstrates that the performance of MIMO-MRC is tightly coupled with the statistics of $\lambda_{\max }$. We next focus on evaluating the outage probability of this system.

\section{A. Outage Probability}

The outage probability characterizes the quality of service (QoS) provided by the system, and is a more generic performance measure of user experience. It is formally defined as the probability of $\gamma$ falls below a certain threshold value, $\gamma_{\text {th }}$, which determines the minimum SNR level for satisfactory reception. Following Theorem 1, the outage probability can be written as

$$
P_{\text {out }}\left(\frac{\gamma_{\text {th }}}{\bar{\gamma}}\right)=\operatorname{Pr}\left\{\gamma<\gamma_{\text {th }}\right\}=F_{\lambda_{\max }}\left(\frac{\gamma_{\text {th }}}{\bar{\gamma}}\right)
$$

where $F_{\lambda_{\max }}(x)$ is given by (10) with the following reparameterization:

$$
\mu=K(K+1) \operatorname{tr}\left(\mathbf{T}^{-1} \overline{\mathbf{H}}^{\dagger} \overline{\mathbf{H}} \mathbf{T}^{-1}\right), \eta=K \operatorname{tr}\left(\mathbf{T}^{-1} \overline{\mathbf{H}}^{\dagger} \overline{\mathbf{H}}\right),
$$

and $\boldsymbol{\alpha}=\left(\begin{array}{ll}\alpha_{11} & \alpha_{12}\end{array}\right)^{T}$ denotes the leading eigenvector of the rank-1 matrix $\boldsymbol{\Lambda}^{-1} \mathbf{U}^{\dagger} \overline{\mathbf{H}}^{\dagger} \overline{\mathbf{H}} \mathbf{U} \boldsymbol{\Lambda}^{-1}$. Here $\mathbf{U}$ and $\boldsymbol{\Lambda}$ are related to $\mathbf{T}$ through the eigen-decomposition $\mathbf{T}=\mathbf{U} \boldsymbol{\Lambda} \mathbf{U}^{\dagger}$ and $\boldsymbol{\Sigma}=$ $\operatorname{diag}\left(\sigma_{1}, \sigma_{2}\right)=(K+1) \boldsymbol{\Lambda}^{-1}$.

The accuracy of (14) is verified in Fig. 2, which plots the outage probability versus the normalized SNR threshold $\gamma_{\text {th }} / \bar{\gamma}$ for different Rician factors (different $K$ values). For numerical simulation purposes, we have used $\mathbf{T}=\boldsymbol{\Psi}$ and $\overline{\mathbf{H}}=\boldsymbol{\Upsilon}$ matrices given in (11). Note that for these analytical curves, the infinite sum (10) has been truncated to a maximum of 15 terms, thereby demonstrating a fast convergence rate for each case. The figure displays a close match between the simulations and analytical results, which verifies the accuracy of the analytical derivations. A counterintuitive trend is also visible - namely the outage does not uniformly decreases with the strength of the LoS component for all $\bar{\gamma}$ values. In particular, in the large $\bar{\gamma}$ regime (i.e., low $\gamma_{\text {th }} / \bar{\gamma}$ regime), outage improves with the increasing strength of the LoS component. In contrast, the opposite trend can be observed in the low $\bar{\gamma}$ regime. Therefore, when the transmit SNR is high, the outage is benefited by having a strong LoS component, whereas in the low transmit SNR regime, a rich scattering environment certainly helps improve the outage. Thus, from an outage point of view and counterintuitively, it is not always beneficial to have stronger LoS links. 


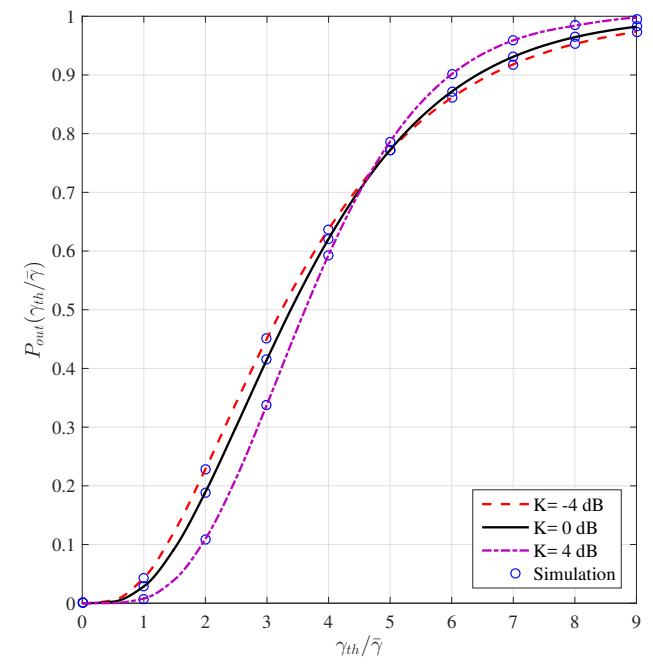

Fig. 2: Outage probability vs normalized SNR threshold $\gamma_{\mathrm{th}} / \bar{\gamma}$ for different $K$ values.

\section{B. The Effect of $K$}

We now investigate the effect of Rician factor $K$ on the outage probability. In particular, we focus on the large $K$ regime. The following proposition characterizes the outage probability as $K \rightarrow \infty$ :

Proposition 1. Let $\overline{\mathbf{H}}^{\dagger} \overline{\mathbf{H}}=4 \mathbf{v} \mathbf{v}^{\dagger}$, where $\|\mathbf{v}\|=1$. Then we have, as $K \rightarrow \infty$

$$
\sqrt{\frac{K}{8 \bar{\gamma}^{2} \mathbf{v}^{\dagger} \mathbf{T} \mathbf{v}}}(\gamma-4 \bar{\gamma}) \stackrel{\mathcal{D}}{\rightarrow} \mathcal{N}(0,1)
$$

where $\stackrel{\mathcal{D}}{\rightarrow} \mathcal{N}(0,1)$ denotes the convergence in distribution to a standard normal random variable.

Proof. Omitted due to space limitation, Please see [1].

An immediate consequence of Proposition 1 is

$$
\lim _{K \rightarrow \infty} \operatorname{Pr}\left\{\sqrt{\frac{K}{8}}(\gamma-4 \bar{\gamma}) \leq \gamma_{\text {th }}\right\}=\Phi\left(\frac{\gamma_{\text {th }} / \bar{\gamma}}{\sqrt{\mathbf{v}^{\dagger} \mathbf{T} \mathbf{v}}}\right)
$$

where $\Phi(z)$ is the c.d.f of the standard normal random variable. Clearly, once properly centered and scaled, the asymptotic outage depends on the channel mean and correlation through the positive definite quadratic form $\mathbf{v}^{\dagger} \mathbf{T} \mathbf{v}$. It is noteworthy that this quadratic form depends on $\mathbf{T}$ but not its inverse. Therefore, the asymptotic outage expression remains valid even when $\mathbf{T}$ is rank deficient. Since we are interested in outage probability, we may use (16) to approximately characterize it, for sufficiently large $K$ values, as

$$
P_{\text {out }}^{\text {Large } K}\left(\frac{\gamma_{\text {th }}}{\bar{\gamma}}\right) \approx \Phi\left(\frac{\gamma_{\text {th }} / \bar{\gamma}-4}{\sqrt{8 \mathbf{v}^{\dagger} \mathbf{T v} / K}}\right) .
$$

It is interesting to observe that the parameter $K$ is completely decoupled from the other parameters in (17).

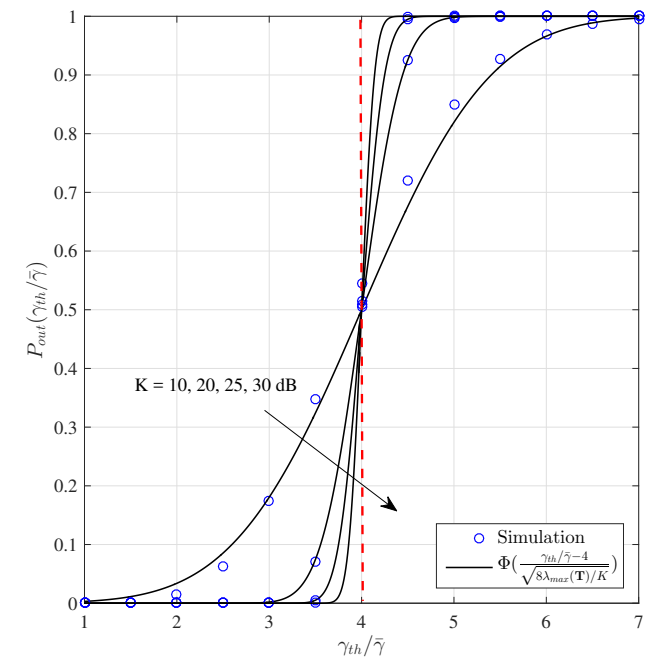

Fig. 3: Formation of an outage barrier at $\gamma_{\mathrm{th}} / \bar{\gamma}=4$.

The effect of large $K$ on the outage probability is depicted in Fig. 3. As we already know, for $K \rightarrow \infty$, the maximum eigenvalue tends to concentrate around 4 (i.e, $\gamma$ around $4 \bar{\gamma}$ ). Therefore, as the figure shows, the outage curves bend more sharply at the critical point and ultimately converges to the vertical barrier at normalized SNR of 4 .

\section{CONCLUSION}

This paper has focused on characterizing $2 \times 2$ MIMOMRC system over correlated Rician fading and a strong LoS component (rank-1 mean) with respect to outage probability. We first derive a new expression for the c.d.f. of the maximum eigenvalue of a $2 \times 2$ correlated non-central Wishart matrix with rank-1 non-centrality parameter. This expression, which in turn facilitates the derivation of the outage, contains a fast converging infinite series of functions. Our analysis demonstrates that as the Rician factor grows large, the outage can be approximately characterized by the c.d.f. of a certain Gaussian random variable. Interestingly, our analysis shows that a strong LoS path is not always beneficial from the outage perspective.

\section{APPENDIX A \\ PROOF OF THEOREM 1}

Here we provide an outline of the main proof due to space limitations.

For clarity let us recall (9)

$$
Q_{k}(x)=\int_{\mathbf{0} \prec \mathbf{Y} \prec \mathbf{I}_{2}} \operatorname{etr}(-x \boldsymbol{\Sigma} \mathbf{Y})\left(\boldsymbol{\alpha}^{\dagger} \mathbf{Y} \boldsymbol{\alpha}\right)^{k} \mathrm{~d} \mathbf{Y} .
$$

Clearly, this integral is performed over the space of Hermitian positive definite matrices $\mathbf{Y} \in \mathbb{C}^{2 \times 2}$ such that $\mathbf{I}_{2}-\mathbf{Y} \succ \mathbf{0}$. This more complicated integral region in turn makes the above matrix integral intractable with the available tabulated results in the literature. Therefore, we now take a closer look at this 
particular integral region. To this end we first parameterize the Hermitian positive definite matrix $\mathbf{Y}$ as

$$
\mathbf{Y}=\left(\begin{array}{ll}
y_{11} & y_{12} \\
y_{12}^{*} & y_{22}
\end{array}\right)
$$

where $y_{11}, y_{22}>0, y_{12} \in \mathbb{C}$ and $y_{11} y_{22}-\left|y_{12}\right|^{2}>0$. Since we require $\mathbf{I}_{2}-\mathbf{Y} \succ \mathbf{0}$ to be satisfied, the above parameters should also fulfill

$$
\left(1-y_{11}\right)\left(1-y_{22}\right)-\left|y_{12}\right|^{2}>0, \quad y_{11}, y_{22} \in(0,1) .
$$

Now keeping in mind the representation $y_{12}=\Re y_{12}+\mathfrak{i} \Im y_{12}$, we can combine the above results to write the integration region corresponding to $\mathbf{0} \prec \mathbf{Y} \prec \quad \mathbf{I}_{2}$ as $\mathcal{R}=\mathcal{R}_{1} \cup \mathcal{R}_{2}$ where

$$
\begin{gathered}
\mathcal{R}_{1}=\left\{\left(y_{11}, y_{22}, \Re y_{12}, \Im y_{12}\right): y_{11}+y_{22}<1,\right. \\
\left.\left|y_{12}\right|^{2}<y_{11} y_{22}, y_{11}, y_{22} \in(0,1)\right\} \\
\mathcal{R}_{2}=\left\{\left(y_{11}, y_{22}, \Re y_{12}, \Im y_{12}\right): y_{11}+y_{22}>1,\right. \\
\left.\left|y_{12}\right|^{2}<\left(1-y_{11}\right)\left(1-y_{22}\right), y_{11}, y_{22} \in(0,1)\right\} .
\end{gathered}
$$

Capitalizing on the above facts we can simplify the matrix integral in (18) to yield the scalar form

$$
Q_{k}(x)=P_{1}(k, x)+P_{2}(k, x)
$$

where

$$
\begin{aligned}
P_{1}(k, x)= & \int_{\mathcal{R}_{1}} \exp \left(-x \sigma_{1} y_{11}-x \sigma_{2} y_{22}\right) \\
& \times\left(\left|\alpha_{11}\right|^{2} y_{11}+\left|\alpha_{12}\right|^{2} y_{22}+2 \Re\left[\alpha_{11}^{*} \alpha_{12} y_{12}\right]\right)^{k} \mathrm{~d} \mathbf{Y} \\
P_{2}(k, x)= & \int_{\mathcal{R}_{2}} \exp \left(-x \sigma_{1} y_{11}-x \sigma_{2} y_{22}\right) \\
& \times\left(\left|\alpha_{11}\right|^{2} y_{11}+\left|\alpha_{12}\right|^{2} y_{22}+2 \Re\left[\alpha_{11}^{*} \alpha_{12} y_{12}\right]\right)^{k} \mathrm{~d} \mathbf{Y}
\end{aligned}
$$

and we have used the differential relation $\mathrm{d} \mathbf{Y}=$ $\mathrm{d} y_{11} \mathrm{~d} y_{22} \mathrm{~d} \Re y_{12} \mathrm{~d} \Im y_{12}$. To facilitate further analysis, we make use of the polar transformation, $\mathrm{d} \Re y_{12}=r \cos \phi$ and $\mathrm{d} \Im y_{12}=$ $r \sin \phi$ with $\mathrm{d} \Re y_{12} \mathrm{~d} \Im y_{12}=r \mathrm{~d} r \mathrm{~d} \phi$, and evaluate the resultant four-dimensional integrals (i.e., with respect to $y_{11}, y_{22}, r$ and $\phi)$ to arrive at the final answer

\section{REFERENCES}

[1] P. Dharmawansa, K. Kahatapitiya, S. Atapattu, and C. Tellambura, "Outage analysis of $2 \times 2$ MIMO-MRC in correlated Rician fading," Available: https://arxiv.org/abs/1811.06712.

[2] L. Zheng and D. N. C. Tse, "Diversity and multiplexing: a fundamental tradeoff in multiple-antenna channels," IEEE Trans. Inform. Theory, vol. 49, no. 5, pp. 1073-1096, May 2003.

[3] T. L. Marzetta, "Noncooperative cellular wireless with unlimited numbers of base station antennas," IEEE Trans. Wireless Commun., vol. 9, no. 11 , pp. 3590-3600, Nov. 2010.

[4] S. Atapattu, P. Dharmawansa, C. Tellambura, and J. Evans, "Exact outage analysis of multiple-user downlink with MIMO matched-filter precoding," IEEE Commun. Lett., vol. 21, no. 12, pp. 2754-2757, Dec. 2017.

[5] S. Atapattu, Y. Jing, H. Jiang, and C. Tellambura, "Relay selection and performance analysis in multiple-user networks," IEEE J. Select. Areas Commun., vol. 31, no. 8, pp. 1517-1529, Aug. 2013.

[6] H. Q. Ngo, A. Ashikhmin, H. Yang, E. G. Larsson, and T. L. Marzetta, "Cell-free massive MIMO versus small cells," IEEE Trans. Wireless Commun., vol. 16, no. 3, pp. 1834-1850, Mar. 2017.
[7] A. Diab, Self-Organized Mobile Communication Technologies and Techniques for Network Optimization, 1st ed. Hershey, PA, USA: IGI Global, 2016.

[8] P. A. Dighe, R. K. Mallik, and S. S. Jamuar, "Analysis of transmitreceive diversity in Rayleigh fading," IEEE Trans. Commun., vol. 51, no. 4, pp. 694-703, 2003.

[9] B. D. Rao and M. Yan, "Performance of maximal ratio transmission with two receive antennas," IEEE Trans. Commun., vol. 51, no. 6, pp. 894-895, Jun. 2003.

[10] M. R. McKay, A. J. Grant, and I. B. Collings, "Performance analysis of MIMO-MRC in double-correlated Rayleigh environments," IEEE Trans. Commun., vol. 55, no. 3, pp. 497-507, 2007.

[11] S. Jin, M. R. McKay, X. Gao, and I. B. Collings, "MIMO multichannel beamforming: SER and outage using new eigenvalue distributions of complex noncentral Wishart matrices," IEEE Trans. Commun., vol. 56, no. 3, 2008.

[12] Y. Wu, R. H. Louie, and M. R. McKay, "Asymptotic outage probability of MIMO-MRC systems in double-correlated Rician environments," IEEE Trans. Wireless Commun., vol. 15, no. 1, pp. 367-376, 2016.

[13] T. Ratnarajah, "Topics in complex random matrices and information theory," Ph.D. dissertation, University of Ottawa (Canada), 2003.

[14] G. Fraidenraich, O. Leveque, and J. M. Cioff, "On the MIMO channel capacity for the Nakagami- $m$ channel," IEEE Trans. Inform. Theory, vol. 54, no. 8, pp. 3752-3757, Aug. 2008.

[15] N. Auguin, D. Morales-Jimenez, and M. R. McKay, "Exact statistical characterization of $2 \times 2$ Gram matrices with arbitrary variance profile," IEEE Trans. Veh. Technol., vol. 66, no. 9, pp. 8575-8579, Sep. 2017.

[16] L. Moreno-Pozas, D. Morales-Jimenez, M. R. McKay, and E. MartosNaya, "Largest eigenvalue distribution of noncircularly-symmetric Wishart-type matrices with application to Hoyt-faded MIMO communications," IEEE Trans. Veh. Technol., vol. 67, no. 3, pp. 2756-2760, Mar. 2018.

[17] M. Charafeddine and A. Paulraj, "Simplified eigenvalues distributions of $2 \times 2$ complex noncentral Wishart," in Asilomar COnf. on Signals, Systems and computers, Nov. 2007, pp. 1215-1218.

[18] R. U. Nabar, H. Bolcskei, and A. J. Paulraj, "Diversity and outage performance in space-time block coded Ricean MIMO channels," IEEE Trans. Wireless Commun., vol. 4, no. 5, pp. 2519-2532, 2005.

[19] M. Kang and M. S. Alouini, "Capacity of MIMO Rician channels," IEEE Trans. Wireless Commun., vol. 5, no. 1, pp. 112-122, Jan. 2006.

[20] P. Dharmawansa and M. R. McKay, "Extreme eigenvalue distributions of some complex correlated non-central Wishart and gamma-Wishart random matrices," J.Multivariate Anal., vol. 102, no. 4, pp. 847-868, 2011.

[21] A. T. James et al., "Distributions of matrix variates and latent roots derived from normal samples," Ann. Math. Statist., vol. 35, no. 2, pp. 475-501, 1964.

[22] A. Davis, "Invariant polynomials with two matrix arguments extending the zonal polynomials: Applications to multivariate distribution theory," Ann. Inst. Statist. Math., vol. 31, no. 1, pp. 465-485, 1979.

[23] _ "On the construction of a class of invariant polynomials in several matrices, extending the zonal polynomials," Ann. Inst. Statist. Math., vol. 33, no. 1, pp. 297-313, 1981 .

[24] P. J. Forrester and T. Nagao, "Eigenvalue statistics of the real Ginibre ensemble," Phys. Rev. Lett., vol. 99, no. 5, p. 050603, 2007.

[25] M. L. Mehta, Random Matrices. Elsevier, 2004, vol. 142.

[26] A. Zanella, M. Chiani, and M. Z. Win, "On the marginal distribution of the eigenvalues of Wishart matrices," IEEE Trans. Commun., vol. 57, no. 4, Apr. 2009.

[27] M. Matthaiou, M. R. McKay, P. J. Smith, and J. A. Nossek, "On the condition number distribution of complex Wishart matrices," IEEE Trans. Commun., vol. 58, no. 6, pp. 1705-1717, Jun. 2010.

[28] A. Constantine, "Some non-central distribution problems in multivariate analysis," Ann. Math. Statist., vol. 34, no. 4, pp. 1270-1285, 1963.

[29] P. Koev and A. Edelman, "The efficient evaluation of the hypergeometric function of a matrix argument," Math. Comp., vol. 75, no. 254, pp. 833846, 2006.

[30] A. M. Mathai, Jacobians of Matrix Transformations and Functions of Matrix Arguments. World Scientific Publishing Company, 1997.

[31] A. Takemura, "Zonal polynomials," Lecture Notes-Monograph Series, vol. 4, pp. 1-104, 1984.

[32] M. Kang and M.-S. Alouini, "Largest eigenvalue of complex Wishart matrices and performance analysis of MIMO MRC systems," IEEE J. Select. Areas Commun., vol. 21, no. 3, pp. 418-426, 2003. 


\section{University Library}

\section{- M M N E R VA A gateway to Melbourne's research publications}

Minerva Access is the Institutional Repository of The University of Melbourne

Author/s:

Dharmawansa, P;Kahatapitiya, K;Atapattu, S;Tellambura, C

Title:

On the Exact Outage Probability of 2×2 MIMO-MRC in Correlated Rician Fading

Date:

2020-06-19

Citation:

Dharmawansa, P., Kahatapitiya, K., Atapattu, S. \& Tellambura, C. (2020). On the Exact

Outage Probability of $2 \times 2$ MIMO-MRC in Correlated Rician Fading. Proceedings of the 2020 IEEE Wireless Communications and Networking Conference (WCNC), 2020-May, IEEE. https://doi.org/10.1109/WCNC45663.2020.9120712.

Persistent Link:

http://hdl.handle.net/11343/241456 